\title{
Emprendimiento familiar inmigrante: el caso de las mujeres chinas en España.
}

\section{Immigrant family business: the Chinese women in Spain}

\author{
Marta Gutiérrez Sastre ${ }^{\mathrm{a}^{*}}$ \\ ${ }^{a}$ Departamento de Sociología y Comunicación, Universidad de Salamanca, Salamanca (España)
}

D A T OS ARTÍC U L O

Historial:

Recibido 27-09-2014

Aceptado 30-10-2014

Palabras clave:

Mujer

Empresa familiar

China

Familia

Inmigración

Códigos JEL:

J16

\footnotetext{
A R T I C LE IN F O

Article history:

Received 27-09-2014

Accepted 05-04-2014

Keywords:

Woman

Family Firms,

China

Family

Migration project

JEL codes:

$\mathrm{J} 16$
}

\begin{abstract}
R E S U M E N
En el marco de un proyecto migratorio condicionado por el emprendimiento familiar, el artículo aborda el desempeño empresarial de las mujeres chinas afincadas en España. Para ello se estudian tres aspectos fundamentales: el significado otorgado al emprendimiento por parte de la comunidad inmigrante china, el valor concedido a la esfera productiva empresarial en relación a la esfera doméstica y el apoyo generado desde las redes familiares transnacionales para el desempeño de responsabilidades empresariales. A través del uso de entrevistas cualitativas a mujeres y hombres chinos residentes en España, se constata el protagonismo de las primeras en el desarrollo de negocios propios, la prevalencia empresarial respecto a las cargas domésticas y la innegable contribución del grupo familiar al desarrollo empresarial de las mujeres chinas.
\end{abstract}

La autora agradece la ayuda recibida del Instituto de la Mujer. Ministerio de Sanidad, Servicios Sociales e Igualdad.

* Autor de contacto.

Correos electrónicos: magusa@usal.es 


\section{Introducción}

Aunque hay constancia de la presencia china en España desde finales del XIX (Beltrán, 2009) su visibilidad no se hizo patente hasta los años de fuerte crecimiento económico (1996-2007). En términos relativos, el colectivo chino no representa más de un $3 \%$ del total de extranjeros, pero es evidente que el impacto económico y laboral de su actividad se coloca muy por encima de esa cifra. Según los datos del Padrón Municipal para 2013, se cuenta con 181.701 empadronados de nacionalidad china, una cifra -por encima de los 27.574 registrados en 2001que constata el crecimiento demográfico constante de esta población en España.

Un alto porcentaje de los inmigrantes chinos residentes en España procede de una misma provincia, la de Zhejiang al sur del país, caracterizada por una cultura emprendedora y una red familiar extensa y trasnacional. Es a este grupo al que se dirige el estudio.

El emprendimiento constituye, por tanto, un rasgo laboral destacado para los hombres y las mujeres chinas de este colectivo. Frente al peso que desempeñan en otros colectivos de mujeres inmigrantes las actividades laborales de cuidado destaca el protagonismo de las mujeres chinas en el desarrollo empresarial. Esta situación, junto a la necesidad de ampliar los estudios sobre un grupo insuficientemente conocido, invita al análisis del caso.

La literatura existente ha demostrado la importancia del grupo étnico y familiar, que act en el desarrollo de negocios propios. Este trabajo se ocupa de valorar el papel que desempeña la red familiar en relación a la actividad empresarial de las mujeres chinas. La pregunta a la que trata de responder este artículo es ¿hasta qué punto la actividad empresarial de las mujeres chinas en España es el resultado de una estrategia de la unidad familiar transnacional?.

Para ello se tendrán en cuenta tres aspectos: el primero, el valor con el que la comunidad china dota al emprendimiento; el segundo, la importancia conferida al campo productivo empresarial (trabajo, negocio, financiación, recursos económicos...,) en relación a las actividades domésticas (familia, roles, tareas de cuidado, relaciones entre géneros...); y por último, el papel que ejerce el entramado familiar transnacional en la actividad empresarial de las mujeres chinas.

El artículo se desarrolla en tres partes bien diferenciadas; una primera en la que se analizan las principales referencias teóricas sobre los negocios familiares de las mujeres inmigrantes en general y de las mujeres chinas en particular. Un segundo apartado constituido por el estudio en sí, donde se explica la metodología utilizada y los resultados en lo que a estas tres dimensiones se refiere (noción de emprendimiento, valor de lo empresarial y lo doméstico y el apoyo familiar en la actividad empresarial). El último apartado, a modo de conclusiones, da respuesta a la pregunta inicial y plantea los términos para la continuidad del trabajo en el futuro.

\section{Emprendimiento familiar y género}

La cuestión de los negocios familiares en los inmigrantes es un tema ampliamente tratado en la literatura norteamericana (Nee y Sanders, 1985, Portes y Bach, 1985; Logan et. al., 1994; Sanders y Nee, 1996; Portes 1997) que ha cobrado fuerza en España a medida que iba creciendo el volumen de población extranjera que desarrollaba estas fórmulas laborales en el país (Beltrán, 2000, 2006; Bodega et. al, 2004; Martínez, 2007; Oso y Ribas, 2006; Solé et. al., 2007).

Desde una perspectiva de género, los negocios familiares han sido motivo de críticas al percibirse como una prolongación de los roles tradicionales de esposa y madre. Estudios internacionales realizados en este sentido concluyen que los proyectos migratorios articulados sobre la base de los negocios familiares se mantienen a costa de la sobrexplotación (Light y Bhachu, 1993) y de la subordinación de las mujeres (Westwood y Bhachu, 1988; Phizacklea, 1988; Anthias y Mehta, 2003). Sin embargo, también se han encontrado evidencias de signo contrario que señalan los aspectos positivos de los negocios familiares a la hora de facilitar la autonomía personal (Morokvasic, 1991), los beneficios directos o una mejor conciliación de la vida familiar y laboral (Song, 1995).

A nivel internacional los estudios sobre el emprendimiento en las mujeres inmigrantes incluyen tanto los factores push, los que expulsan a los inmigrantes del mercado asalariado (Hisrich y Brush, 1984; Phizacklea, 1988; Heilman y Chen, 2003) como los pull, los factores de atracción del mercado hacia la actividad empresarial (Morokvasic, 1991; Mizrachi, 2005). En el particular caso español la idea de "emprendimiento inmigrante como refugio" ha sido bien recibida al considerar el autoempleo como una buena alternativa para aquellos grupos peor valorados en el mercado de trabajo nacional. Antes que como motivación 
intrínseca, el impulso fundamental para la creación de negocios parecería encontrarse en la necesidad de las mujeres inmigrantes por abandonar las posiciones marginales que sufren en la estructura ocupacional. La segmentación étnica aparece el principal motivo para la creación de negocios (Solé y Parella 2005; Gonzalez-Gonzalez et al, 2011).

Por otro lado, estudios de corte culturalista hablan de la existencia de comunidades - como pudieran ser la judía, la pakistaní o la china- en las que existe un comportamiento aprendido hacia el negocio (Sowell, 1996), donde las "minorías intermediarias" (middleman minority) utilizan su pertenencia a un grupo étnico como el capital clave para obtener beneficios comerciales y mejorar en la escala social (Bonacich, 1973). Los trabajos de Portes (Wilson y Portes, 1980; Portes, 1981, 1997) y su desarrollo del concepto de "enclave étnico" explican el valor que adquieren los mecanismos de solidaridad interna que se producen en los grupos de inmigración; mecanismos capaces de facilitar la integración laboral de sus miembros y de generar crecimiento económico en paralelo a los mercados de trabajo nacionales.

\section{El emprendimiento de las mujeres chinas}

El giro acometido por la sociedad china y su encumbramiento como referencia económica mundial constituye uno de los fenómenos más sorprendentes de las últimas décadas. El interés que suscita la relación entre familia china, negocio y desarrollo económico data de antiguo si bien la perspectiva de análisis ha ido variando con el paso del tiempo. Si las relaciones familiares chinas fueron consideradas un obstáculo para la modernización del país, hoy en día, su expansión económica no podría entenderse al margen del impulso empresarial originado desde la unidad familiar (Wong, 1988).

El peso de las mujeres chinas en la aportación familiar también ha ido variando. En los primeros estudios sobre el tema la presencia de las mujeres era insignificante o dirigida a mencionar su papel tradicional y sumiso (Greenhalgh, 1985; King White, 1996), pero en la actualidad, su figura es interpretada como clave en el desarrollo de negocios tanto dentro como fuera del país (Deng et al, 2011).

Con la mirada puesta en la prosperidad familiar, el proyecto migratorio de los chinos de Zhejiang se planifica en origen sobre la base de la familia extensa trasnacional gracias a la cual los migrantes y sus descendientes siguen ligados con su país de origen y con sus redes de parentesco (Schiller, Basch y Blanc, 1994; Saiz López 2012). La familia extensa trasnacional recoge distintas generaciones -abuelos, pero también tíos y primos...- e incluye tanto a los sujetos que emigran como a los que se quedan en China, que también participarían en el proyecto migratorio; todos aportan bienes diferentes y necesarios para la reproducción social de los grupos domésticos (Nieto, 2002). Esta unidad familiar de carácter transnacional se erige como el primero y más básico de sus capitales sociales ya que resulta determinante en el proceso de integración económica al país de destino.

La familia extensa transnacional distribuye funciones claras a cada uno de sus miembros para garantizar la fuerte inversión productiva. La inversión productiva afecta a los sujetos que emigran, que deberán trabajar para conseguir el ascenso social del grupo; a los sujetos que se quedan en China se les encomienda asumir el coste de la reproducción social (Saiz López, 2012), es decir todas las tareas relacionadas con el cuidado del grupo especialmente de los sujetos más dependientes. Solo en el caso en que estas tareas reproductivas no pongan en riesgo las responsabilidades productivas de los que emigran (p. ej. el negocio familiar) podrán llegar a asumirla en la sociedad de destino. Esto significa que la prioridad de las mujeres chinas que migran -lo mismo que la de los hombres- se dirige al plano productivo y sólo asumirán los costes reproductivos cuando este plano esté garantizado.

La inversión empresarial de las mujeres inmigrantes chinas se apega al mantenimiento de la estructura y las expectativas familiares. $\mathrm{Si}$ en entornos occidentales el éxito profesional de las mujeres genera situaciones conflictivas en el terreno familiar, las mujeres chinas parecen no tener que afrontar ese tipo de tensiones (Saiz López, 2005; Pérez-Cerezo, 2009). Fuera de interpretaciones personales o políticas sobre el significado que tienen sus triunfos laborales, las mujeres chinas asocian el éxito empresarial con el éxito en la familia y en el matrimonio (Deng et al. 2011). De la misma forma, las referencias sobre la naturalidad con la que las mujeres chinas desarrollan su proyecto empresarial con negocios familiares en los que la mujer desempeña un papel principal o en igualdad de condiciones con su marido (Ip et al. 1999), son la evidencia del reconocimiento familiar a las actividades productivas de sus miembros, sean hombres o mujeres. 
Existen referencias sobre el desigual valor que tradicionalmente se ha asignado a las mujeres y a los hombres -afiliación patrilineal, obligación del pago de la dote (Botton Beja 1993, 1995)- y sobre la subordinación de la mujer china a la esfera reproductiva (Nieto, 2007, Lee et. al, 2002). Pero también cabe pensar en su reconocimiento social a través del ejercicio de una actividad laboral vinculada a los negocios. En este sentido, el propio hecho migratorio podría acentuar la oportunidad de reconocimiento para estas mujeres.

Investigaciones internacionales que valoran la experiencia de la inmigración china en Occidente (Preston y Man 1999; Zhou 2000, 2004; Man 2004) evalúan el desaliento que provoca en estas mujeres el desempleo, la precariedad y la falta de reconocimiento laboral. Si conseguir un trabajo remunerado es fundamental para el mantenimiento de la unidad familiar, la actividad empresarial se muestra como una de las mejores fórmulas para ser socialmente reconocidas.

\section{Metodología del estudio}

La investigación se ha servido de metodología cualitativa por entender que era la que mejor facilitaba el acercamiento a las comunidades inmigrantes y al tema objeto de estudio (Gubrium y Holstein, 1997). Las particularidades de la comunidad estudiada, con situaciones legales y socioeconómicas diversas y un conocimiento limitado del castellano aconsejaban una aproximación pausada, personal y flexible al entorno y a las problemáticas sobre las que los sujetos desarrollan su vida cotidiana.

La técnica de investigación utilizada en el estudio fue la entrevista personal semiestructurada que permitió percibir las trayectorias personales de los entrevistados y los procesos de toma de decisiones efectuados en el marco familiar. Se descartó el uso de técnicas grupales (grupos de discusión) por las dificultades lingüísticas que presentaba para los sujetos hablar en un contexto de grupo y para evitar (posibles) presiones por parte del grupo de coétnicos a la hora de expresar opiniones, valoraciones o experiencias.

Este artículo procede de una investigación más amplia centrada en estudiar las condiciones de vida y trabajo de las mujeres asiáticas en su conjunto, pero aquí se extraen los materiales procedentes del colectivo chino únicamente. El guión seguido en las entrevistas se centraba en conocer las expectativas asociadas al proyecto migratorio, las condiciones sociolaborales, el valor del trabajo y el negocio y el significado de las relaciones familiares. Siguiendo el principio de saturación para determinar el tamaño de la muestra, se realizaron un total de 28 entrevistas a miembros de la comunidad china. De cara a asegurar una mayor heterogeneidad en los perfiles de los entrevistados y a incluir en el estudio diversas redes coétnicas, el trabajo de campo se llevó a cabo en distintas ciudades de la geografía española - en concreto Alicante, Asturias, Comunidad de Madrid, Valencia, y Salamanca- , durante los meses de Octubre de 2009 a Mayo de 2010.

El que la investigación se centrara en el estudio de las mujeres chinas propició que fueran más mujeres que hombres las entrevistadas y que éstas tuvieran en su conjunto un marcado perfil emprendedor aunque también se incluyeron mujeres empleadas por cuenta ajena y otras dos que aun no habían comenzado a trabajar. Además se realizaron siete entrevistas a varones chinos para conocer su discurso respecto al valor del trabajo, el emprendimiento y respecto al papel que tienen las mujeres en cuanto a las tareas productivas $\mathrm{y}$ domésticas.

El perfil de todos los inmigrantes entrevistados cumple con los siguientes tres requisitos: a) personas nacidas en China mayores de 15 años, b) que llevaran al menos 6 años residiendo en España y c) que tuvieran un conocimiento suficiente del español como para mantener una conversación fluida en la entrevista. Aunque se trató de que las entrevistas se llevaran a cabo en lugares que permitieran cierta intimidad, la mayor parte de ellas se efectuaron en el propio lugar de trabajo (bazar, restaurante, tienda de ropa, kiosko...). Se acordó con los entrevistados un momento del día en que o bien el negocio estaba cerrado o no contaba con gente o había otra persona encargada del mismo para que la entrevistada/o no tuviera que abandonar la entrevista para atender a la clientela. Las entrevistas que no se realizaron en el lugar de trabajo se realizaron en cafeterías o en el domicilio de la entrevistada/o. Las sesiones fueron grabadas digitalmente con el permiso de los entrevistados y posteriormente transcritas literalmente.

Es necesario mencionar la buena acogida por parte de los entrevistados a participar en la investigación y a ser grabados. Esta predisposición pudo deberse a la forma en la que se llevó a cabo el contacto, nunca directamente sino a través de informantes que conocían a miembros de la comunidad china. Los informantes procedían tanto de redes formales (asociaciones de inmigrantes, 
Tabla 1

Perfil de las personas entrevistadas.

\begin{tabular}{|c|c|c|c|c|}
\hline Perfil & Edad & Estado civil & Años en España & Actividad Laboral \\
\hline Mujeres chinas 21 & 22-52 años & $\begin{array}{l}\text { Casadas } 16 \\
\text { Solteras } 5\end{array}$ & $\begin{array}{l}\text { Más de } 10 \text { años: } 5 \\
8 \text {-10 años: } 11 \\
\text { Menos de } 8 \text { años: } 5\end{array}$ & $\begin{array}{l}\text { Estudiantes } 1 \\
\text { Cuenta propia } 15 \\
\text { Cuenta ajena } 4 \\
\text { No trabajan } 1\end{array}$ \\
\hline Hombres chinos 7 & 16-35 años & $\begin{array}{l}\text { Casados } 3 \\
\text { Solteros } 4\end{array}$ & $\begin{array}{l}\text { Más de } 10 \text { años: } 1 \\
8-10 \text { años: } 3 \\
\text { Menos de } 8 \text { años: } 3\end{array}$ & $\begin{array}{l}\text { Cuenta propia } 4 \\
\text { Estudiantes } 3\end{array}$ \\
\hline
\end{tabular}

cámaras de comercio, instituciones públicas, servicios sociales) como informales (amigos y conocidos que tuvieran relaciones de confianza con inmigrantes chinos). También se utilizó la técnica de la bola de nieve para poder identificar, a partir de los propios entrevistados, a otros sujetos que cumplieran con los criterios de inclusión.

El uso de informantes podría haber generado sesgos en los perfiles de los entrevistados. Estos informantes procedían de distintos lugares geográficos $\mathrm{y}$ diversas posiciones sociales pero todos eran españoles con contactos en la comunidad china. Por otro lado, la estrategia de la bola de nieve trató de reducir esa sobrerrepresentación de chinas y chinos con "relaciones de confianza con personas españolas", su objetivo era el de avanzar en la investigación dentro de la propia comunidad china ya que, en este caso, la persona de contacto tenía origen chino. Tanto el uso de informantes españoles como la técnica de la bola de nieve se realizaron para evitar recelos respecto a los motivos de la entrevista y la grabación y es probable que sin su uso, algunas de las entrevistas no hubieran podido llevarse a cabo. Cabe reflexionar, no obstante, sobre las limitaciones de este trabajo a la hora de extrapolar resultados de la comunidad china en su conjunto. Aunque este artículo no pretende ninguna generalización estadística de sus conclusiones, los resultados que se presentan sí permiten abrir vías de investigación respecto a una comunidad relevante pero aún poco conocida en España.

Además se realizaron dos entrevistas a expertas en la comunidad china; se trata de dos mediadoras socioculturales. La investigación está orientada a conocer la perspectiva china de la relación entre trabajo, negocio y familia, por lo que sus aportaciones no han sido tenidas en cuenta para las conclusiones de este artículo, sin embargo, hay que reconocer que el discurso de estas técnicas sí permitió avanzar en un mayor conocimiento de la comunidad. El hecho de que hubieran vivido en China durante años y hablaran chino sirvió de puente cultural a la hora de facilitar la comprensión sobre valores o expectativas de la comunidad que resultaban desconocidas pero importantes de cara a los objetivos planteados del trabajo. De esta forma, el sentido de estas entrevistas era acercar al equipo de investigación el significado de las prácticas, recursos y problemáticas de la comunidad china en su estancia en España.

\section{Empresa familiar para la comunidad china en España}

El hecho de que alrededor de un 70\% de los inmigrantes chinos residentes en España proceda de una misma provincia, Zhejiang, confiere al grupo una alta homogeneidad. Zhejiang cuenta con una larga trayectoria de emigración hacia Europa y un impulso empresarial ha dado lugar a una marca particular, el "espíritu de Zhejiang" (Zhejiang spirit) (Beltrán, 2006) caracterizado por el deseo unánime de éxito económico a través de un trabajo duro y del desarrollo de negocios.

El espíritu de Zhejiang ayudaría a explicar la predisposición de buena parte de los residentes chinos por el desarrollo de negocios propios. Las diferencias que les separan del otro gran grupo de residentes chinos, los chinos del norteste familiarizados con el trabajo asalariado y carentes de red familiar en España (Saiz López, 2012), nos advierten de la necesidad de subrayar las diferentes estrategias, recursos y expectativas seguidos por cada uno de los grupos.

En España la relación de la comunidad del sur de China con el emprendimiento ha sido estudiada por varios autores (Beltrán 2000, 2004; Beltrán et. al, 2006, Cachón, 2005; 2007; Nieto, 2002; Saiz López 2005) que señalan no sólo el papel determinante del grupo doméstico en la marcha del negocio, sino también su eficacia en la diversificación comercial, su dispersión geográfica y las ventajas comerciales que obtienen gracias a la flexibilidad legislativa española y al peso de la economía sumergida. Todos estos factores, unidos a los efectos laborales de las políticas migratorias y a la fuerte estratificación étnica del mercado productivo español (Cachón, 
2009), explicarían los motivos de la especialización laboral del colectivo chino.

Respecto a otros colectivos de inmigrantes, la comunidad china destaca por su carácter emprendedor y su elevada tasa de empleo $\left(77^{\prime} 5 \%\right.$ según datos de la EPA frente al 53'6\% del total de población extranjera para el año 2010). Los datos del Anuario Estadístico de la Inmigración revelaban que, para 2009, el 38\% del total de los trabajadores y trabajadoras chinos afiliados a la Seguridad Social trabajaban por cuenta propia, mientras que para el total de los trabajadores extranjeros las cifras se situaban en el $13^{\prime} 24 \%$ y en el $18^{\prime} 5 \%$ en el caso de los trabajadores españoles. Como dato curioso destaca que para ese mismo año, las mujeres chinas alcanzaban cifras algo superiores a las de sus compatriotas varones situándose en el $40^{\prime} 19 \%$ de trabajadoras por cuenta ajena frente al $36^{\prime} 43 \%$ de los trabajadores chinos.

El motivo principal de la inmigración china a España es el factor económico; las entrevistadas y entrevistados hablan del desplazamiento migratorio como un proyecto dirigido a "ganar dinero", "mejorar la vida", "enriquecerse" para luego poder regresar a su país de origen y vivir mejor. Las redes familiares se desarrollan sin un especial arraigo al territorio concibiendo como temporal su presencia en cualquiera de las localidades de residencia. No en vano es el grupo de inmigrantes que presenta mayores tasas de movilidad interna dentro de la geografía española $(17,9 \%$ frente al 3’9\% según Carrasco y García, 2011).

La homogeneidad de la migración china procedente del sur (con personas mayoritariamente ubicadas en el comercio y la hostelería, una red familiar extensa y transnacional, bajos niveles de cualificación, unas similares aspiraciones de éxito económico...) facilita el seguimiento de unas trayectorias sociolaborales internas muy parecidas entre sí. En lo que afecta a las mujeres, y al margen de su edad o estado civil, comienzan su andadura en el país trabajando durante jornadas laborales que pueden superar las 14 horas diarias en negocios propios, o en los de algún miembro de la familia extensa. Hasta el momento, la incorporación de las mujeres chinas al mercado laboral español ha sido inmediata, siendo las propias redes familiares las responsables de garantizar amparo legal y recursos económicos desde el primer momento.

La política migratoria española de apoyo a la reagrupación familiar ha resultado muy conveniente para el conjunto chino ya que ha permitido culminar un proyecto migratorio concebido desde sus orígenes como familiar y basado en la incorporación laboral plena de todos sus miembros. Si para otras comunidades inmigrantes la reagrupación familiar de las mujeres como "esposas" no llega a garantizarles actividad laboral alguna, para la mujer china es el grupo familiar el que proporciona y tramita su participación laboral activa.

Aunque la entrada al mercado laboral de las mujeres chinas en España sea como empleadas en negocios ajenos, la motivación hacia el emprendimiento se mantiene. Es frecuente aspirar a la creación de un negocio familiar propio confiando en que, con el tiempo, y a través del matrimonio, el ahorro y el préstamo interno, esto pueda ser posible. El emprendimiento se considera el camino natural para asegurar la prosperidad familiar y el hecho de no haber arriesgado o de seguir trabajando como empleado para otros es interpretado en términos negativos por parte del conjunto. Se piensa que con el tiempo se ha de intentar iniciar un negocio, aunque no siempre prospere. La visión aquí es similar para los hombres y para las mujeres pues ambos comparten el deseo de participar del éxito económico

Emprender un negocio es una aspiración de todo el que llega y no alcanzarlo puede considerarse una pequeña derrota, sobre todo entre aquellos que llevan más años en España. Las mujeres como miembros activos de la estructura familiar asumen de manera generalizada el riesgo de montar su propio negocio - diferente del de su esposo-o de abanderar uno de carácter familiar. El haber vivido experiencias de emprendimiento en la familia facilita la asunción del papel de emprendedoras. Son constantes los ejemplos de mujeres que a lo largo del tiempo han emprendido varios negocios, casi siempre distintos pues consideran que la diversificación comercial es una clave del éxito empresarial.

Para montar un negocio se requiere contar con inversión económica y capital humano suficiente, pero las familias no escatiman a la hora de destinar recursos al emprendimiento de las nuevas generaciones sean estas hombres o mujeres. Un negocio es la mejor muestra de éxito y prestigio familiar por lo que todo ahorro dedicado al establecimiento del mismo se da por bien empleado.

El vínculo matrimonial entreteje lazos afectivos y alianzas económicas. La familia actúa como una empresa cuando genera y gestiona recursos económicos, de igual forma que la empresa funciona sobre la base de unos valores y prácticas compartidas. Las relaciones de confianza, esfuerzo y 
compromiso son imprescindibles y la demanda de lealtad es una constante.

En el marco de la empresa familiar, la unidad de los miembros de la familia se erige como el ente básico para la supervivencia reproductiva y económica pero, a su vez, la esfera productiva, que materializa el proyecto migratorio en la sociedad de destino, sirve para crear y fortalecer lazos de parentesco retroalimentando las redes familiares de apoyo. En las parejas jóvenes que vinieron solteros a España, los vínculos matrimoniales están muy vinculados al trabajo lo que fortalece la seguridad de la relación. Por otro lado, el hecho de que las redes laborales sean grandes permite contar con mayor variabilidad a la hora de poder elegir pareja sin hacer reducir por ello el referente de la confianza como condición indispensable.

El proyecto migratorio chino no puede mantener una distribución de roles que aparte a las mujeres de la esfera laboral. Se observa la subordinación de lo reproductivo, ubicado siempre en un segundo plano respecto a la hegemonía productiva-empresarial, y se reconocen formas que evidencian la división sexual del trabajo (con tareas que suelen hacer los hombres, y tareas que suelen hacer las mujeres; contabilidad del negocio para estas últimas por ejemplo), aunque esta división se aleja de la tradicional dicotomía de lo productivo como lo masculino versus lo reproductivo/domestico como lo femenino.

El objetivo familiar de maximizar beneficios para el grupo desaprueba los intentos personales de independencia y éxito individual y lleva a que el emprendimiento femenino sea interpretado en términos familiares

Pero la unidad familiar extensa no es sólo una cuestión que afecte a las relaciones de género, también se incluye la colaboración intergeneracional. El carácter intergeneracional del proyecto migratorio chino abarca tanto "a los que se van" como "a los que se quedan", y se hace evidente en relación al papel de los abuelos y a su implicación activa en los costes de reproducción social. La crianza de los nietos pequeños por parte de los abuelos u otro familiar (primos, tíos ...) en China, es una situación muy frecuente que permite el máximo desarrollo productivo de las generaciones laboralmente activas. No existe conflicto entre trabajo y cuidado de los hijos ya que se considera que la estrategia permitirá reforzar las relaciones familiares, los hijos estarán bien criados, y podrán socializarse en la cultura y en el idioma chino. Cuando los hijos son ya mayores, su presencia en los centros de trabajos no genera problemas, lo que borra la frontera entre familia y trabajo y refleja la búsqueda de responsabilidades compartidas también entre los más jóvenes.

Esta práctica no afecta a todos los grupos de inmigrantes chinos por igual ya que son las familias menos favorecidas económicamente, o los recién llegados, los que con más facilidad la llevan a cabo. Las familias que tienen negocio propio son las que mejor pueden asumir la crianza de sus hijos en España - contratando algún pariente o coétnico para que ayude en la tarea- sin necesidad de enviarlos a China.

El componente económico del vínculo familiar se aprecia en la relación con los hijos. El trabajo de los adultos se entiende dirigido no sólo a garantizar la subsistencia de los menores sino a facilitarles su desarrollo posterior, en este sentido destaca la importancia del "legado económico" y el patrimonio. La delegación del cuidado directo a otros familiares es una forma de asegurar desde la distancia el futuro de los descendientes. Se trabaja para los hijos aunque no sea a través del cuidado directo con la idea de que, con el tiempo, el dinero acumulado les permita establecer $s u$ propio negocio.

\section{Algunos datos concluyentes: El valor del negocio familiar}

En el caso de la comunidad china afincada en España, el valor de la empresa familiar y su proyecto migratorio se concreta en los siguientes aspectos:

\subsection{Emprendimiento familiar chino como forma de vida.}

En el marco de la comunidad china estudiada el emprendimiento empresarial constituye la esencia de su proyecto migratorio; éste se percibe como una inversión necesaria para la mejora social, que es, en definitiva, el objetivo de su desplazamiento. El trabajo asalariado, cuando no se trata de una etapa temporal encaminada al ahorro para un futuro negocio, se vive con frustración y, aunque no todos llegan a establecer un negocio, el emprendimiento constituye un marco de referencia frecuente. A diferencia de otras comunidades de inmigrantes en las que el emprendimiento sí podría ser un refugio a la falta de integración en el mercado de trabajo nacional, para los chinos procedentes del sur de China la creación de empresas propias representa la esencia de la movilidad, el progreso y el reconocimiento social. Los proyectos empresariales que emprenden las mujeres chinas no difieren de los 
proyectos de los varones y la intensidad con la que éstas afrontan la actividad empresarial es notoria. El apoyo familiar dirigido a fortalecer su inversión empresarial es una evidencia de su reconocimiento.

\subsection{Reconocimiento en clave familiar de los éxitos productivos de las mujeres.}

La fuerte inversión productiva de las mujeres chinas en España forma parte de una estrategia familiar que trata de maximizar los recursos disponibles priorizando el desarrollo laboral de todos sus miembros activos. En línea con la importancia concedida a esta esfera productiva, el éxito empresarial de la mujer se percibe como una mejora en el nivel de vida familiar y, aunque no rompe con la subordinación de género, sí implica su reconocimiento social. La propiedad jurídica de una empresa por parte de las mujeres chinas no garantizaría un control personal y único sobre la actividad empresarial pero es motivo de reconocimiento social.

\subsection{Asunción de funciones y responsabilidades por parte de la familia extensa}

La predisposición de la familia extensa transnacional hacia la cobertura de las necesidades del grupo redime a la mujer de las funciones reproductivas en exclusividad. Las mujeres trabajarían en el ámbito doméstico de manera indirecta y a través de una "hiperinversión" productiva-empresarial que trata de garantizar la seguridad económica de los descendientes (por ejemplo con el pago de bienes, el ahorro para la educación o para una futura inversión empresarial de los hijos, el envío de paquetes al país de origen..).

\section{Conclusiones}

Si los conceptos de "enclave étnico" y "minorías intermedias" resultan fundamentales para conocer el emprendimiento inmigrante en su conjunto, para comprender el caso de las mujeres chinas es necesario analizar las relaciones que se generan en la unidad familiar.

En el emprendimiento chino apenas hay espacio para el desarrollo de trayectorias individuales, pero el grupo familiar garantiza un fuerte apoyo hacia los esfuerzos empresariales realizados tanto por mujeres como por hombres. Este apoyo se manifiesta sobre la base de tres mecanismos: un aparataje económico (un sistema de préstamos que facilita la creación de negocios a hombres y a mujeres), un aparataje simbólico (por el cual las figuras emprendedoras, sean mujeres $\mathrm{u}$ hombres, gozan de un mayor prestigio y status social) y, por último, una organización del cuidado, que beneficia especialmente a las mujeres empresarias y que se manifiesta en la forma por la cual la unidad familiar extensa transnacional trabaja para reducir el coste reproductivo de los miembros más activos, cuidando por ejemplo a los hijos para facilitar las responsabilidades productivas de los sujetos laboralmente activos.

Desde una perspectiva de género la proyección productiva de las mujeres chinas no permite hablar de relaciones en términos de mayor equidad. El reconocimiento del trabajo de las mujeres, más que al desarrollo de aspiraciones individuales, parece encontrarse en la base de una concepción familiar muy apegada al marco productivo. En este sentido, el estudio sí permite presentar el contexto y las relaciones que se producen en el seno de los proyectos migratorios determinados por el emprendimiento familiar.

\section{Bibliografía}

Anthias, F. y Mehta, N. (2003). Intersection between gender, the family, and self-employment: The family as a resource. International Review of Sociology, 13, 105-16.

Beltrán, J. (2000). La empresa familiar. Trabajo, redes sociales y familia en el colectivo chino. Ofrim/Suplementos, 6, 129-153.

Beltrán, J. (2004). Las comunidades chinas en España y sus actividades económicas. Economía Exterior, 30, 153-160.

Beltrán, J. (2006). El empresariado como modo de vida. El caso de los inmigrantes chinos. En J. Beltrán, et al. (eds.), Empresariado étnico en España, Madrid, Observatorio Permanente de la Inmigración (OPI), Ministerio de Trabajo y Asuntos Sociales, Fundación CIDOB, 231-248.

Beltrán, J (2009). La presencia china en España: Globalización y transnacionalismo, en Fundació Seminario de Investigación para la Paz, ed., La emergencia de China e India en el siglo XXI. Colección Actas, 72, Serie "Estudios para la Paz", 23. Zaragoza, Gobierno de Aragón, 249-271.

Beltrán, J., Oso, L. y Ribas, N. (2006). Un campo de estudio para el empresariado étnico en España. En, J. Beltrán, L. Oso, y N. Ribas, (coords.), Empresariado étnico en España, Madrid: Fundació CIDOB/Observatorio Permanente de la Inmigración. 
Bodega, M.I., Cebrián de Miguel, J. A., Borbonado, J. (2004). La iniciativa empresarial del inmigrante;

Economistas, 99, 116-123.

Bonacich, E. (1973). A Theory of Middleman Minorities, American Sociological Review, 38, 583-594.

Botton Beja, F. (1993). La familia en la sociedad tradicional. En Botton Beja, Bajo Un mismo techo: la familia tradicional en China y su crisis, México: Colegio de Méjico.

Botton Beja, F. (1995). Familia y cambio social en China, Revista de Occidente, 172, 51-64.

Cachón, L. (2005). Bases sociales de los sucesos de Elche de septiembre de 2004. Crisis industrial, inmigración y xenofobia. Madrid: Observatorio Permanente de la Inmigración.

Cachón, L. (2009). La "España inmigrante": marco discriminatorio, mercado de trabajo y políticas de integración. Barcelona, Anthropos

Carrasco, C. y García C. (2011). Inmigración y mercado de trabajo. Documentos del Observatorio Permanente de la Inmigración. Informe 2011, Madrid: Observatorio Permanente de la Inmigración.

Deng, S., Xu W. y Ilan A. (2011). Framework for female entrepreneurship in China. International Journal of Business and Emerging Markets 3 (1), 3-20.

González-González, J., Bretones, F., Zazco, V., y Andrés, R. (2011). Women, Immigration and Entrepreneurship in Spain: A Confluence of Debates in the Face of a Complex Reality. Women's Studies International Forum, 34, 360-370.

Greenhalgh, S. (1985). Sexual Stratification: The Other Side of "Growth with Equity" in East Asia, Population and Development Review, 11, (2), 265-314.

Gubrium, J. y Holstein, J. (1997). The New Language of Qualitative Method. Oxford, Oxford University Press.

Heilman, M. y Chen, J. (2003). Entrepreneurship as a Solution: the Allure of Self-Employment for Women and Minorities, Human Resource Management Review, 13 (2), 347-364.

Hisrich, R y Brush, C. (1984). The Women Entrepreneur: Management Skills and Business Problems, Journal of Small Business Management, 22 (1), 30-37.

Ip, D. y Lever- Tracy, C. (1999). Asian women in business in Australia. En G. Kelson \& D. DeLaet (Ed.), Gender and immigration. Basingstoke: Macmillan Press.

King White, M. (1996). The Chinese Family and Economic Development: Obstacle or Engine?. Economic Development and Cultural Change, 45 (1), 1-30.

Lee, M. Chan, A. Bardby, H. y Green, G. (2002).Chinese migrant women and families in Britain Women's Studies International Forum, 25(6), 607-618.
Li, P.S (1977). Fictive Kinship, Conjugal Tie and Kinship Claim among Chinese Immigrants in the United States, Journal of Comparative Family Studies 8(1), 4764.

Light, I. y Bhachu, P. (1993). Immigration and Entrepreneurship: Culture, Capital, and Ethnic Networks. New Brunswick: Transaction Publishers.

Logan, J., Alba, R. y McNulty, T. (1994). Ethnic Economies in Metropolitan Regions, Miami and beyond, Social Forces, 72, 691-724.

Man, G. (2004). Gender, work and migration: Deskilling Chinese immigrant women in Canada, Women's Studies International Forum, 27, (2), 135-148

Martínez, P. (2007). Empresas familiares de inmigrantes, Papers, 85, 229- 231.

Mizrachi, B. (2005). The Henna Maker: A Moroccan Immigrant Woman Entrepreneur in an Ethnic Revival. En L. A. Keister (ed.), Entrepreneurship, 15, Emerald Group Publishing Limited, 257-277

Morokvasic, M. (1991). Roads to Independence: Selfemployed Immigrants and Minority Women in Five European States, International Migration Review, 19(3), 407-420.

Nee, V. y Sanders, J. (1985). The Road to Parity: Determinants of the Socioeconomic Achievements of Asian Americans.Ethnic and Racial Studies 8, 75-93.

Nieto, G. (2002). La emigración de Qingtian a España. Reflexiones desde una perspectiva de género. En A. García-Mina, y M.J. Carrasco, Cuestiones de género en el fenómeno de las migraciones, Universidad Pontificia de Comillas.

Nieto, G. (2007). La inmigración china en España: una comunidad ligada a su nación, Madrid: Catarata.

Oso, L. y Ribas, N. (2006). Empresariado étnico y relaciones de género: mujeres dominicanas y marroquíes en Madrid y Barcelona. En J. Beltrán, L. Oso y N. Ribas, N. (coords.), Empresariado étnico en España, Madrid: Fundació CIDOB/Observatorio Permanente de la Inmigración.

Pérez-Cerezo, J. (2009). Emprendedoras chinas, Madrid: Lid.

Phizacklea, A. (1988). Entrepreneurship, ethnicity and gender. En S. Westwood y P. Bhachu (eds), Enterprising Women: ethnicity, economy and gender relations, London: Routledge, 20-34.

Portes, A. (1981). Modes of Structural Incorporation and Present Theories of Labor Immigrations. En M.M. Kritz, C.B. Keely, and S.M. Tomasi (eds), Global Trends in Migration. Staten Island, NY, CMS Press.

Portes, A. (1997). Globalization from Below: The Rise of Transnational Communities, Princeton: Princeton University 
Portes, A. y Bach, R. (1985). Latin Journey. Cuban and Mexican Immigrants in the United States, Berkeley: University of California Press.

Preston, V. y Man, G. (1999). Employment Experiences of Chinese Immigrant Women: An Exploration of diversity. Canadian Woman Studies/Les cahiers de la femme, 19(3), 115-122.

Saiz López, A. (2005). Mujeres empresarias chinas en un contexto migratorio: adaptación y continuidad. En Checa y Olmos, Mujeres en el camino: el fenómeno de la migración femenina en España, Barcelona: Icaria.

Saiz López, A. (2012). Mujeres chinas en España. El capital social y su impacto en las estrategias productivas y reproductivas, Papers, 97 (3), 591-612

Sanders, J. y Nee, V. (1996). Immigrant SelfEmployment: The Family as Social Capital and the Value of Human Capital, American Sociological Review, 61(2), 231-249.

Schiller N. Basch, L. y Blanc, C. (1995). From Immigrant to transmigrant: Theorizing transnational migration, Anthropological quaterly, 68 (1), 48-63.

Solé, C. y Parella, S. (2005). Negocios étnicos: los comercios de los inmigrantes no comunitarios en Cataluña, Barcelona, Fundació Cidob,

Solé, C., Parella, S., Cavalcanti, L. (2007). Empresariado inmigrante en España, Barcelona, Fundació La Caixa
Song, M. (1995). Between "the front" and "the back", Chinese Women's Work in Family Businesses. Women's Studies International Forum, 18, (3), 285-298.

Sowell, T. (1996). Migration and Culture: A World View. New York: Basic Books,

Westwood, S. y Bhachu, P. (1988). Enterprising women: ethnicity, economy and gender relations. London: Routledge.

Wilson, K. y Portes, A. (1980). Immigrant enclaves: an analysis of the labor market experiences of Cubans in Miami. American Journal of Sociology, 86 (2), 295-319.

Wong, S.-L. (1988). The Applicability of Asian Family Values to Other Sociocultural Settings. En P. Berger y H- H. Michael Hsiao (eds.) Search of an East Asian Development Model, New Brunswick: Transaction Books.

Zhou, Y. (2000). The fall of "the other half of the sky"? Chinese immigrant women in the New York area, Women's Studies International Forum, 23, (4), 445-459.

Zhou, Y. (2004). Chinese immigrants in the global economy. En M. Toro-Morn y M. Alicea, Migration: A global view, Greenwood's World View of Social Issues Series. 